\title{
Feasibility of generalised diffusion kurtosis imaging approach for brain glioma grading
}

\author{
E. L. Pogosbekian ${ }^{1,2}$ • I. N. Pronin ${ }^{1}$ • N. E. Zakharova ${ }^{1}$ • A. I. Batalov ${ }^{1}$ • A. M. Turkin ${ }^{1}$ - T. A. Konakova ${ }^{1}$ • \\ I. I. Maximov $3,4,5$ (D)
}

Received: 18 September 2020 / Accepted: 23 November 2020 / Published online: 7 January 2021

(C) The Author(s) 2021

\begin{abstract}
Purpose An accurate differentiation of brain glioma grade constitutes an important clinical issue. Powerful non-invasive approach based on diffusion MRI has already demonstrated its feasibility in glioma grade stratification. However, the conventional diffusion tensor (DTI) and kurtosis imaging (DKI) demonstrated moderate sensitivity and performance in glioma grading. In the present work, we apply generalised DKI (gDKI) approach in order to assess its diagnostic accuracy and potential application in glioma grading.

Methods Diffusion scalar metrics were obtained from 50 patients with different glioma grades confirmed by histological tests following biopsy or surgery. All patients were divided into two groups with low- and high-grade gliomas as grade II versus grades III and IV, respectively. For a comparison, trained radiologists segmented the brain tissue into three regions with solid tumour, oedema, and normal appearing white matter. For each region, we estimated the conventional and gDKI metrics including DTI maps.

Results We found high correlations between DKI and gDKI metrics in high-grade glioma. Further, gDKI metrics enabled introduction of a complementary measure for glioma differentiation based on correlations between the conventional and generalised approaches. Both conventional and generalised DKI metrics showed quantitative maps of tumour heterogeneity and oedema behaviour. gDKI approach demonstrated largely similar sensitivity and specificity in low-high glioma differentiation as in the case of conventional DKI method.

Conclusion The generalised diffusion kurtosis imaging enables differentiation of low- and high-grade gliomas at the same level as the conventional DKI. Additionally, gDKI exhibited higher sensitivity to tumour heterogeneity and tissue contrast between tumour and healthy tissue and, thus, may contribute as a complementary source of information on tumour differentiation.
\end{abstract}

Keywords Glioblastoma · Glioma discrimination · Diffusion kurtosis imaging

I. I. Maximov

ivan.maximov@psykologi.uio.no

1 Neuroimaging Department, N.N. Burdenko National Medical Research Centre of Neurosurgery, Moscow, Russian Federation

2 General and Clinical Neurophysiology Lab, Institute of Higher Nervous Activity and Neurophysiology of RAS, Moscow, Russian Federation

3 Department of Psychology, University of Oslo, Oslo, Norway

4 Department of Mental Health and Addiction, Norwegian Centre for Mental Disorders Research (NORMENT), Oslo University Hospital, Oslo, Norway

5 Department of Health and Functioning, Western Norway University of Applied Sciences, Bergen, Norway

\section{Introduction}

Primary brain glioma is a highly widespread type of intraaxial brain tumours seen approximately in one-fourth diagnosed tumour cases [1]. Classification of the brain tumours is performed in accordance with grades introduced by the World Health Organization (WHO) [2] which combine histopathological and molecular features into integrated tumour characterisation. As a result, glioma grades are ordered from grades I up to IV related to tumour's aggressiveness and malignancy. Both the tumour's diagnosis and its grade can be reliably confirmed by a histopathologic analysis of the tumour's tissue obtained from an invasive procedure such as biopsy or surgery. However, non-invasive assessments of the tumour's 
malignancy are necessary for clinical treatment, surgery planning, and survival rate estimations. In particular, this applies to low-grade tumour cases where such assessments contribute to treatment efficacy and, as a result, improve quality of life for patients. Magnetic resonance imaging (MRI) offers a wide spectrum of tissue visualisation approaches for clinical purposes allowing one to detect and localise brain's abnormalities with a certain degree of specificity. Nevertheless, conventional MRI techniques such as structural $T_{1} / T_{2}$-weighted imaging with or without contrast agents or magnetic resonance spectroscopy demonstrate limited sensitivity and specificity for brain glioma differentiation [3, 4].

Diffusion-weighted imaging (DWI) has exhibited its superiority as a non-invasive brain imaging technique and has been applied in a plethora of clinical settings [5-7]. Diffusion tensor imaging (DTI) [8], the most often used DWI approach, has also been utilised for tumour assessment by many research groups [9-11]. In turn, diffusion kurtosis imaging (DKI) [12] is a powerful extension of the conventional DTI technique enabling estimation of the degree of non-Gaussian diffusion in the brain tissue by estimating higher order cumulant of the diffusion signal expansion [13]. The deviation of water molecule diffusion from free diffusion behaviour or Gaussian distribution is caused by complex organisation of the brain tissue, where cellular and neurite barriers alter the probability distribution of water diffusion. This phenomenon is particularly important for the brain tumour detection and assessment due to increased tissue complexity resulting from the high cancer cell proliferation rate, increased tumour tissue vascularisation, presence of oedema, as well as necrosis. DKI is extensively used in brain imaging [14-16], in particular, for tumour differentiation [17-21]. Despite the fact that DKI demonstrated quite promising results in the case of glioma grading, the kurtosis scalar metrics, such as mean kurtosis, still lack the accurate and reliable glioma grading in the case of both low- (i.e. glioma-I and II) and high(glioma-III and IV) grade gliomas [18, 22, 23].

In the present study, we adapted the generalised kurtosis approach (GK) [24] for glioma differentiation in order to evaluate as well as to add a complementary information and, thus, increase the accuracy of glioma grading. An advantage of the third-order term of cumulant expansion of the diffusion signal has been demonstrated for differentiation of low- and highgrade gliomas together with an application of the conventional kurtosis (CK) approach. In order to emphasise the advantage of the DKI imaging for glioma grading, we concurrently applied both DKI and DTI metrics derived from the CK and GK approaches. Hence, the purpose of the present study was to investigate the value of the generalised DKI metrics for the evaluation of glioma grading.

\section{Materials and methods}

\section{Patients}

Fifty adult patients (mean age, $44 \pm 13$ years; minimal age, 24 years; maximal age, 70 years; male/female ratio, 30/20) initially diagnosed with glioma were recruited at our Institution and examined with MRI prior to biopsy, surgery, radiation or chemotherapy treatments. The diagnosis of glioma and the WHO grade [25] were confirmed by histological and immunohistochemical examinations for each subject following MRI examination. Complementary to the histological glioma grade evaluation, the immunohistochemical marker Ki-67 was extracted from pathology using commercial monoclonal antibody. Immunohistochemistry of Ki-67 was performed and retrospectively analysed by a pathologist blinded to the clinical- and MRI-derived information. The Ki-67 rates were estimated by the percentile of immunoreactive cells from 1000 malignant cells. The estimation was performed in the areas with the highest number of positive nuclei within the solid tumour.

The study included 19 patients with glioma grade II, 8 patients with glioma grade III, and 23 patients with glioma grade IV. The group of patients with glioma-II consisted of 6 subjects with oligoastrocytoma, 1 subject with oligodendroglioma, 1 subject with gemistocytic astrocytoma, and 11 subjects with diffuse astrocytoma. The group of patients with glioma-III consisted of 7 subjects with anaplastic astrocytoma and 1 subject with anaplastic oligoastrocytoma. The group of patients with glioma-IV consisted of the 22 subjects with glioblastoma and 1 subject with gliosarcoma.

\section{Imaging}

All patients underwent MRI examination with a $3 \mathrm{~T}$ GE scanner (Signa HDxt) using an 8-channel phased-array head coil. The imaging protocol included precontrast $T_{2}$-weighted FLAIR and diffusion-weighted sequences, followed by postcontrast $T_{1}$-weighted imaging with a gadolinium-based contrast agent. The parameters specific to anatomical imaging sequences were the following: $T_{1}$-weighted: $\mathrm{FOV}=240 \mathrm{~mm}^{2}$, TE/TR $=3500 / 8800 \mathrm{~ms}$, resolution $1 \times 1 \times 1 \mathrm{~mm}^{3} ; T_{2}$-weighted FLAIR: $\mathrm{FOV}=240 \mathrm{~mm}^{2}$, TE/TR $=120 / 9500 \mathrm{~ms}$, resolution $1 \times 1 \times 5 \mathrm{~mm}^{3}$; diffusion-weighted imaging: $\mathrm{FOV}=$ $240 \mathrm{~mm}^{2}, \mathrm{TE} / \mathrm{TR}=102.8 / 10000 \mathrm{~ms}$, resolution $3 \times 3 \times$ $3 \mathrm{~mm}^{3}, b$ values $=0,1000$, and $2500 \mathrm{~s} / \mathrm{mm}^{2}$ with 60 encoding diffusion directions per each $b$-shell; directions were noncoplanar uniformly distributed over the unit sphere. The diffusion imaging was obtained using a single-shot echo planar imaging sequence. 


\section{Image analysis}

Prior to estimation of scalar diffusion metrics, the raw diffusion data were corrected for image distortions in accordance with optimised pipeline [26], i.e. for noise [27] and Gibbsringing distortions [28], eddy-current distortions including bulk head motion artefacts were corrected using eddy utility from FSL [29-31]. Conventional DKI metrics such as mean, axial, radial kurtosis, and kurtosis anisotropy (MK, AK, RK, and KA, respectively) were estimated using an iterative weighted least squares approach [32] and supplied by the complementary DTI measures known as fraction anisotropy (FA), mean diffusivity (MD), axial diffusivity (AD), and radial diffusivity (RD). The conventional DKI metrics were obtained from the second-order cumulant expansion in the following form [12]:

$\ln [S(b)]=\ln \left[S_{0}\right]-\mathrm{bD}+\mathrm{b}^{2} D^{2} \frac{K}{6}=\mathrm{F}_{1}$,

where $b$ is the diffusion weighting, $D$ is the diffusion coefficient, $S_{0}$ is the signal without the diffusion weightings, and $K$ is the diffusion kurtosis. The right-hand expression is defined as $F_{1}$. The generalised DKI parameters were estimated using in-house MATLAB scripts (The MathWorks, Natick, MA, USA) including the third-order terms [24]: a

$\mathrm{R}$

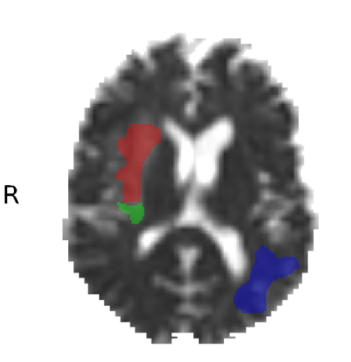

e

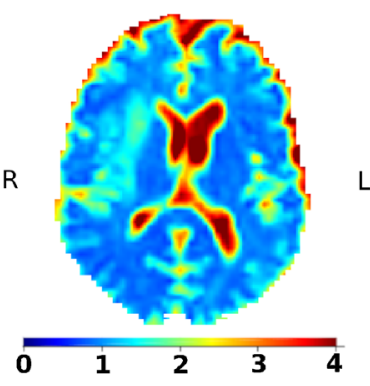

b

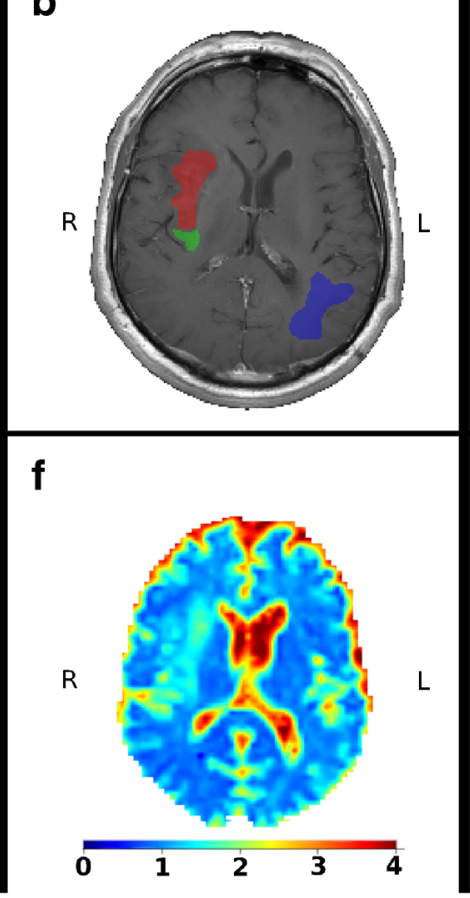

Fig. 1 Example of low-grade gliomas with the marked regions of interests: red is the tumour, green is the oedema, and blue is the NAWM. All maps are already aligned and interpolated to $T_{1}$-weighted image. a mean diffusivity map with masks; $\mathbf{b} T_{1}$-weighted image with contrast agent; $\mathbf{c}$ $T_{2}$-weighted FLAIR image with contrast agent; d $T_{2}$-weighted $\ln [S(\mathrm{~b}, \alpha)]=\mathrm{F}_{1}+(\alpha-1) \cdot D^{3} b^{3} \frac{K^{2}}{54}$

where $\alpha$ is the adjusting variable. In the case of $\alpha=1$, the last equation leads to the conventional DKI expression $F_{1}$. In the present work, we used an optimised $\alpha$ value at 2/7 [24]. Subsequently, the estimated diffusion scalar maps for both cases of conventional and generalised kurtosis were aligned and interpolated to $T_{1}$-weighted anatomical image for each patient using the cubic spline interpolation algorithm and affine transformation. Two independent radiologists manually delineated three regions of interest for each subject: tumour, oedema, and normal appearing white matter (NAWM) using 3 MRI contrasts: $T_{1}$-weighted, $T_{2}$-weighted FLAIR, and MD map estimated from CK approach. Examples of images for low- and high-grade gliomas with the 3 aforementioned regions of interest are presented in Figs. 1 and 2, respectively.

\section{Statistical analysis}

Statistical analyses were performed using $R$ Project (http://rproject.org) and in-house MATLAB scripts. Some voxels among the patient maps contained of corrupted diffusion metrics derived from the DTI and DKI tensors. In order to prevent any statistical bias in analysis, we excluded such voxels from

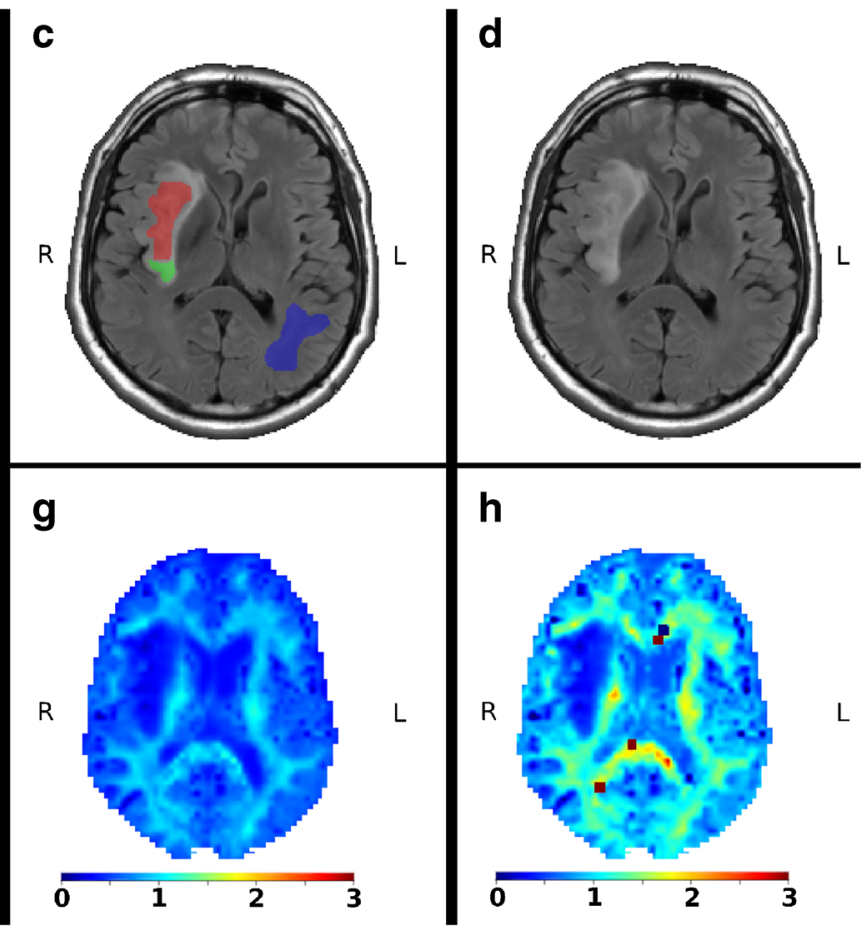

FLAIR image without contrast agent; e mean diffusivity map obtained from the conventional kurtosis approach; f mean diffusivity map obtained from the generalised kurtosis approach; $\mathbf{g}$ mean kurtosis map obtained from the conventional kurtosis approach; and $\mathbf{h}$ mean kurtosis map obtained from the generalised kurtosis approach 


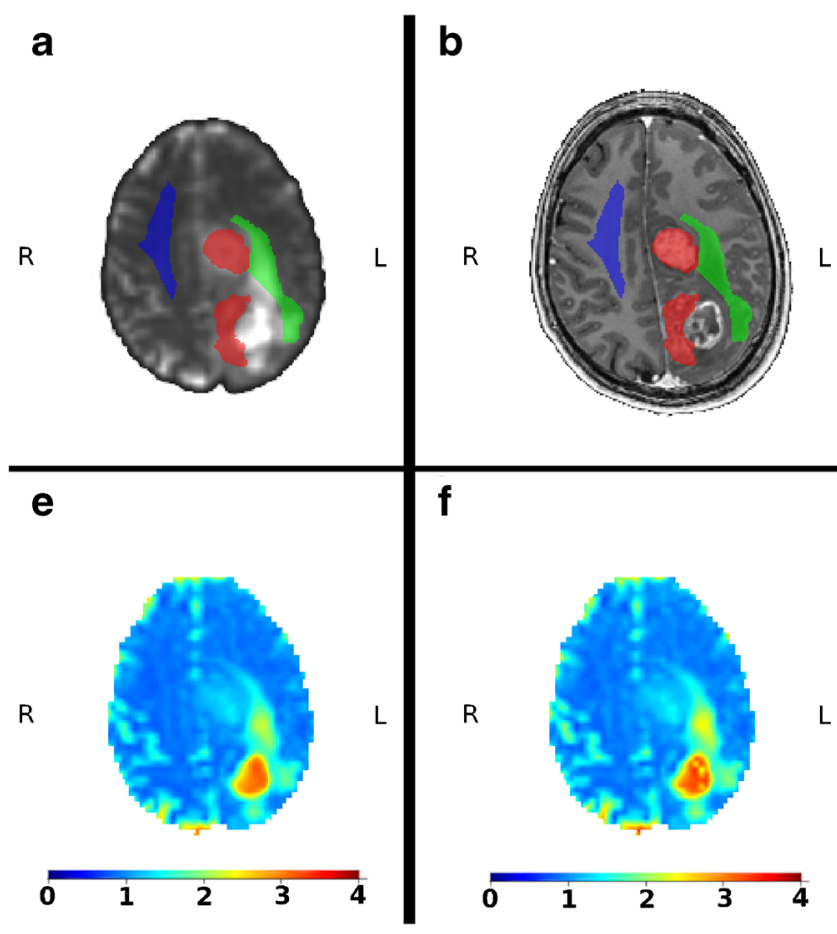

Fig. 2 Example of high-grade gliomas with the marked regions of interests: red is the tumour, green is the oedema, and blue is the NAWM. All maps are already aligned and interpolated to $T_{1}$-weighted image. a mean diffusivity map with masks; $\mathbf{b} T_{1}$-weighted image with contrast agent; $\mathbf{c}$ $T_{2}$-weighted FLAIR image with contrast agent; d $T_{2}$-weighted

consideration using inequalities: $\mathrm{MK}<0$ and $\mathrm{MK}>3$, for both CK and GK approaches. Mean values and standard deviations of all diffusion metrics were calculated from the tumour, oedema, and NAWM regions. These values were used for the low- and high-grade glioma differentiation and assessed with a Mann-Whitney-Wilcox $U$ test with statistical significance reported at the 0.05 level. Effect sizes were calculated with Cohen's $d$ in order to estimate the magnitude of the differences between the conditions/groups. Correlations between the conventional and generalised DKI metrics have been estimated using a linear regression model: $y=k_{0}+k_{1} \bullet x$ and package stat from $R$ Project. Receiver operating c

d

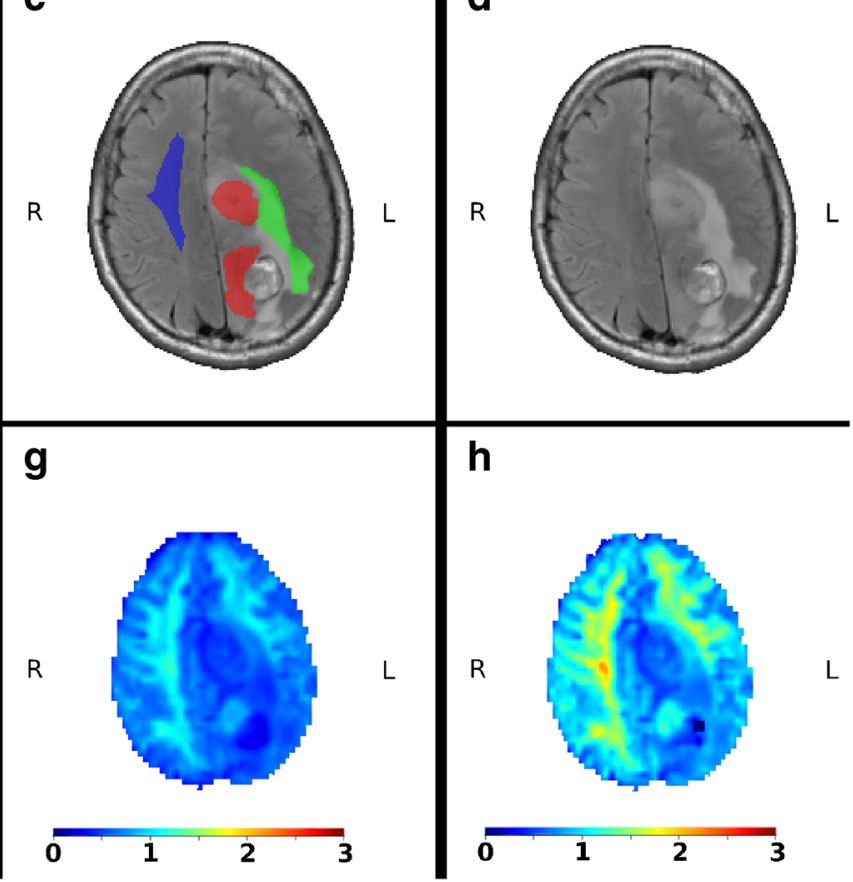

FLAIR image without contrast agent; e mean diffusivity map obtained from the conventional kurtosis approach; $\mathbf{f}$ mean diffusivity map obtained from the generalised kurtosis approach; $\mathbf{g}$ mean kurtosis map obtained from the conventional kurtosis approach; and $\mathbf{h}$ mean kurtosis map obtained from the generalised kurtosis approach

characteristic (ROC) analysis was performed using package $p R O C$ in order to compute the area under curve (AUC) for estimation of the diffusion model performance for tumour differentiation. The squared Pearson correlation coefficients were estimated using the package stat from $R$ Project. In order to estimate a rate of the solid tumour heterogeneity using diffusion metrics derived from CK and GK approaches, we used a root-mean-square estimator (RMSE). The RMSE rate demonstrates the root-mean-square deviation of the diffusion metric from the tumour mean value averaged over the tumour region, i.e. the higher RMSE rate exhibits a higher metric dispersion or heterogeneity.
Table 1 The results for linear correlations between diffusion metrics obtained from $\mathrm{CK}$ and GK approaches (see Figs. 3 and 4)

\begin{tabular}{lllllllll}
\hline & FA & MD & AD & RD & KA & MK & AK & RK \\
\hline LGG & & & & & & & & \\
$\quad$ Intercept $b_{0}$ & 0.053 & -0.017 & 0.091 & 0.025 & 0.19 & -0.12 & 0.0088 & -0.15 \\
$\quad$ Slope $b_{1}$ & 0.73 & 1.1 & 1.0 & 1.0 & 0.88 & 1.7 & 1.7 & 1.6 \\
$\quad$-square & 0.693 & 0.981 & 0.906 & 0.97 & 0.463 & 0.944 & 0.777 & 0.942 \\
HGG & & & & & & & & \\
Intercept $b_{0}$ & 0.028 & 0.022 & 0.054 & 0.028 & 0.14 & -0.1 & -0.018 & -0.13 \\
Slope $b_{1}$ & 0.85 & 1.0 & 1.0 & 1.0 & 0.93 & 1.6 & 1.7 & 1.6 \\
$R$-square & 0.814 & 0.977 & 0.953 & 0.97 & 0.756 & 0.889 & 0.804 & 0.855 \\
\hline
\end{tabular}

The linear model is $y=b_{0}+b_{1} \cdot x$; R-square is the coefficient of determination also coinciding with the squared Pearson correlation coefficient. $L G G$ low-grade glioma, $H G G$ high-grade glioma 

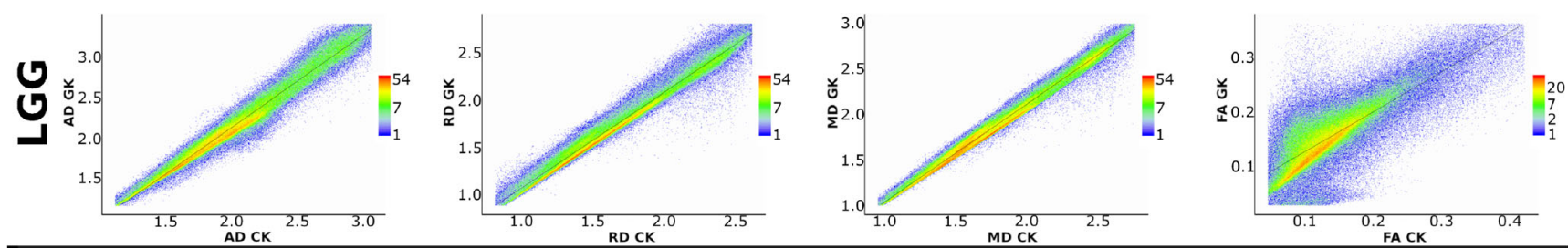
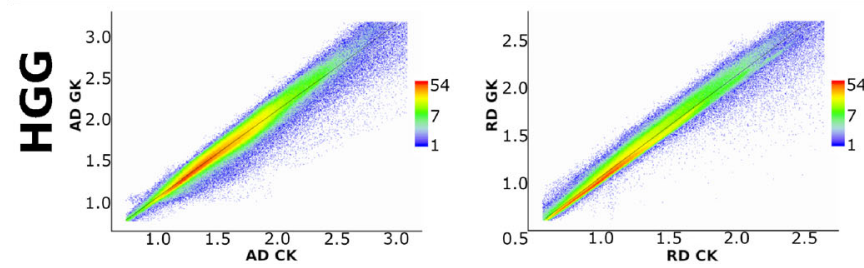

Fig. 3 Voxel-wise correlations of the diffusion tensor metrics between the conventional and generalised kurtosis approaches. The scalar metrics belong to the tumour masks in low- and high-grade gliomas for all

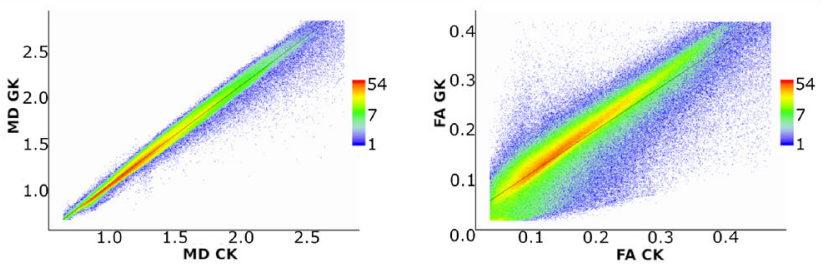

patients. The columns of plot are axial, radial, and mean diffusivities and fractional anisotropy, respectively

\section{Results}

Figures 1 and 2 are visual examples of manual masks of three regions, i.e. tumour, oedema, and NAWM together with examples of MD and MK maps for the low- and high-grade glioma patients, estimated by the CK and GK approaches. All maps are presented in $T_{1}$-weighted space with a spatial interpolation of diffusion maps. Briefly, we can see that the visual quality of MD maps between $\mathrm{CK}$ and GK approaches is very similar. In turn, as already was shown in [24], the tissue contrast for DKI maps, e.g. MK, is higher for GK approach. Squared outlier voxels both in Figs. 1 and 2 are the result of fitting problems due to noisy diffusion signal. These voxels have been excluded from the analysis.

Figures 3 and 4 present the correlations of diffusion metrics obtained by $\mathrm{CK}$ and GK signal expansions. Figure 3 shows DTI metrics only, i.e. FA, MD, AD, and RD, with corresponding R-squared values which in the case of linear correlations coincide with the squared Pearson correlation coefficients (see Table 1). In Fig. 4, we present DKI metrics only, i.e. KA, MK, $\mathrm{AK}$, and RK, with corresponding R-squared values (see Table 1). The scatter plots were estimated voxel-wisely for the tumour masks only from low- and high-grade gliomas, respectively. In short, linear correlations between CK and GK estimations are higher for the high-grade glioma in contrast to the low-grade glioma. This effect is present for all DTI metrics. In the case of DKI metrics, it applies only for KA and AK metrics. Interestingly, MK and RK metrics have two linearly dependent subgroups of voxels (see, e.g. Fig. 4: LGG), which merge as glioma malignancy increases (see, Fig. 4: HGG).

In Fig. 5 we present boxplots of the mask-averaged diffusion metrics estimated from CK and GK approaches. The averages were performed for each ROI such as tumour, oedema, and NAWM for each patient. The boxplots are structured as ROI-wise and glioma grades pairs. For each pair of low-
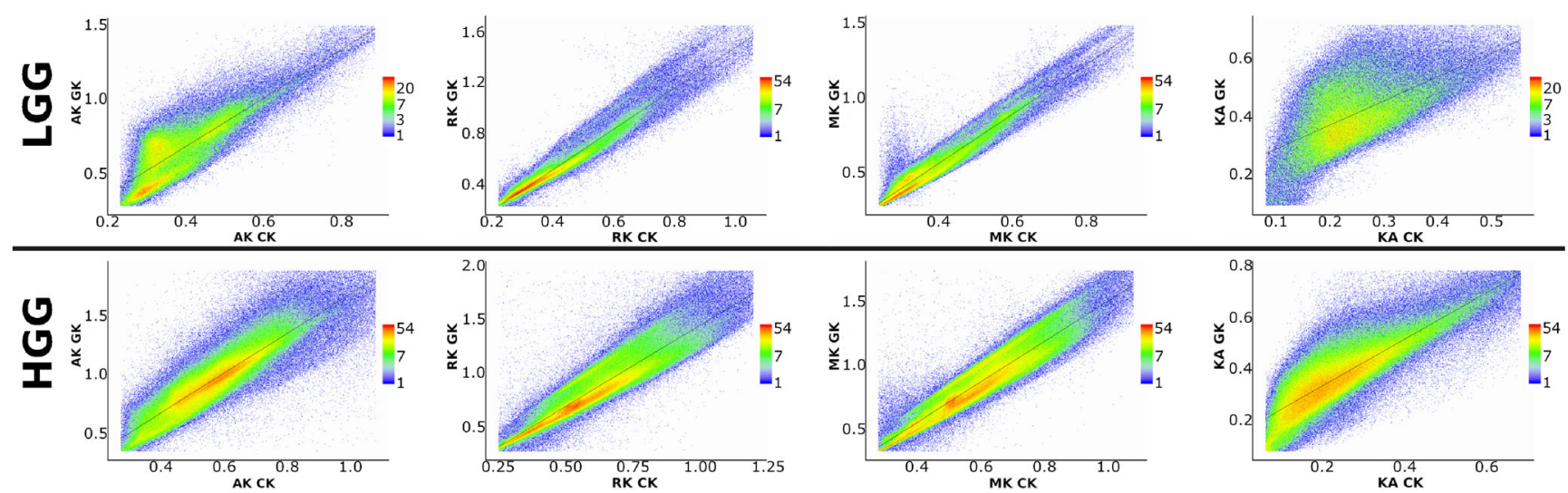

Fig. 4 Voxel-wise correlations of the diffusion kurtosis metrics between the conventional and generalised kurtosis approaches. The scalar metrics belong to the tumour masks in low- and high-grade gliomas for all
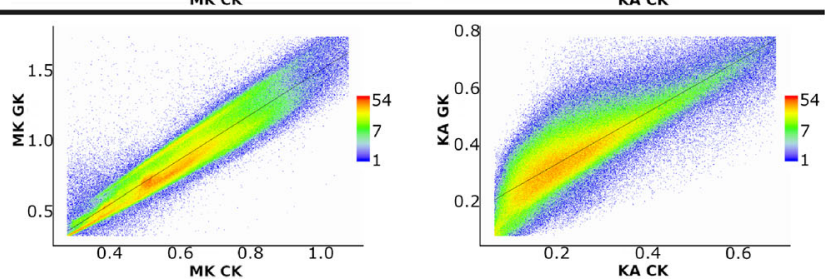

patients. The columns of plot are axial, radial, and mean kurtosis and kurtosis anisotropy, respectively 
Table 2 The effect size of significant differences between low- and high-grade gliomas detected by CK and GK derived diffusion metrics (see Fig. 5)

\begin{tabular}{lllllllll}
\hline $\begin{array}{l}\text { Effect } \\
\text { size }\end{array}$ & FA & MD & AD & RD & KA & MK & AK & RK \\
\hline CK & 0.99 & 1.21 & 1.11 & 1.23 & 0.11 & 1.56 & 1.47 & 1.52 \\
GK & 0.65 & 1.15 & 1.13 & 1.15 & 0.30 & 1.50 & 1.35 & 0.24 \\
\hline
\end{tabular}

The effect size is estimated using the Cohen's $d$

and high-grade gliomas, we performed the Mann-WhitneyWilcox $U$ test in order to check for significant $(p<0.05)$ differences between glioma grades in all regions including tumour, oedema, and NAWM. There were no significant differences between low- and high-grade glioma diffusion metrics for oedema, and there was difference between GK metrics of RD for NAWM. However, for tumour regions, both CK and GK metrics except for KA and FA, we revealed a significant difference for low- and high-grade gliomas (marked by asterisk in Fig. 5). In the case of FA metrics, GK approach did not reveal significant differences between glioma grades. The effect sizes for the diffusion metric differences detected by both CK and GK approaches are summarised in Table 2 . For DTI metrics, the effect size is large for both kurtosis approaches, while for DKI metrics the differentiation possesses very large effect size except for GK-derived RK metric. We also performed a pair comparison between CK and GK metrics marked by the cyan background colour for significant differences $(p<0.05)$ between the CK-GK metrics. Interestingly, for all DKI metrics and ROIs, there were significant difference between CK and GK metrics. However, for DTI metrics, the significant difference was detected only for NAWM regions for FA, MD, and RD metrics.

It is of vital importance to perform a comparison between CK and GK approaches in terms of their sensitivity and specificity. These results are presented in Fig. 6. Both approaches
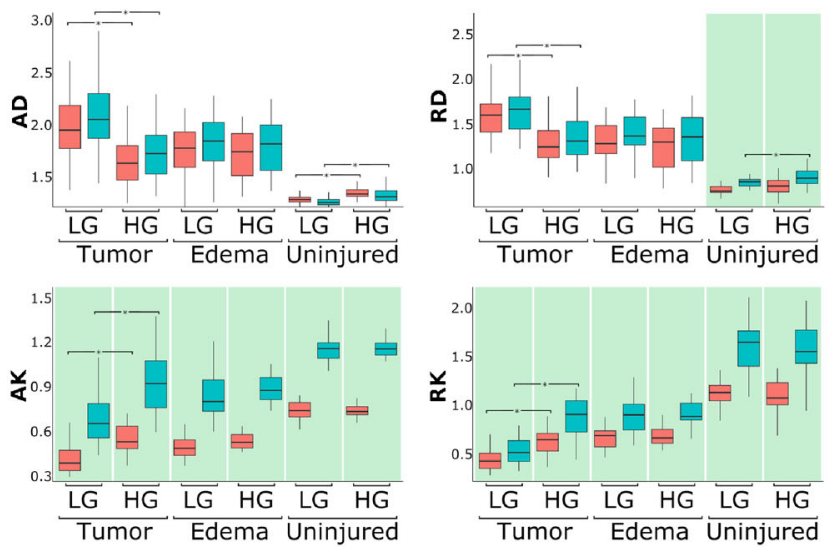

Fig. 5 The boxplots of averaged diffusion tensor and kurtosis metrics in different regions: tumour, oedema, and normally appeared white matter. The star-marked brackets exhibit the significant $(p<0.05)$ difference demonstrated high rate of sensitivity and specificity for low and high glioma discrimination with negligible difference between CK and GK approaches. The estimated sensitivity, specificity, cutoff, and area under curves (AUC) are summarised in Table 3.

In Figs. 7 and 8, we present correlations between Ki-67 and diffusion metrics estimated by CK and GK approaches for DTI and DKI, respectively. The correlations were estimated for all subjects and low- and high-grade subject groups separately. The Spearman's correlation coefficients for diffusion metrics vs Ki-67 are summarised in Table 4. In Figs. 7 and 8, we plotted the linear regressions for low- and high-grade gliomas and performed a quantitative comparison for each approach using cocor function [33]. The significant difference $(p<0.05)$ between LGG and HGG correlations was found only for FA linear coefficients in both $\mathrm{CK}$ and GK approaches.

Figure 9 shows a variability of the diffusion metrics in the solid tumour. The tumour heterogeneity was estimated by RMSE rate obtained for each DKI approach. In order to visualise the tumour heterogeneity, we used the scatterplots for each metrics. Interestingly, the heterogeneity derived from DTI metrics for both approaches does not have the significant difference, i.e. the RMSE points are close to the unity line (see Fig. 9). In turn, the RMSE rates for AK, RK, and MK are higher for GK approach, and the RMSE rate for KA is higher for CK approach. It is worth to note that the heterogeneity estimations obtained by GK approach show higher RMSE rates for both low- and high-grade gliomas.

\section{Discussion}

In the present study, we estimated two diffusion imaging approaches based on the conventional and generalised kurtosis expansions. The scalar metrics based on GK approach
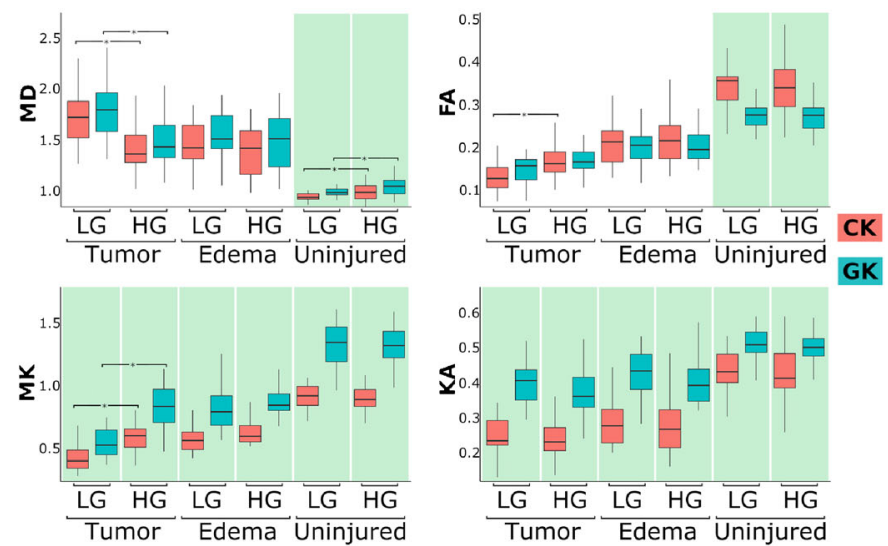

between low- and high-grade gliomas for the diffusion metrics. The green background colour emphasise the significant difference between diffusion metrics for the conventional and generalised approaches 
Table 3 Differentiation performance for diffusion metrics estimated by $\mathrm{CK}$ and GK approaches

\begin{tabular}{lcccccccc}
\hline & FA & MD & AD & RD & KA & MK & AK & RK \\
\hline CK & & & & & & & & \\
AUC & 0.77 & 0.79 & 0.78 & 0.80 & 0.53 & 0.87 & 0.86 & 0.86 \\
Cutoff & 0.15 & 1.50 & 1.72 & 1.39 & 0.23 & 0.53 & 0.41 & 0.54 \\
Sensitivity & 0.74 & 0.71 & 0.71 & 0.71 & 0.48 & 0.74 & 0.93 & 0.74 \\
Specificity & 0.74 & 0.84 & 0.84 & 0.84 & 0.68 & 0.89 & 0.68 & 0.90 \\
GK & & & & & & & & \\
AUC & 0.65 & 0.78 & 0.77 & 0.78 & 0.62 & 0.86 & 0.83 & 0.88 \\
Cutoff & 0.17 & 1.71 & 1.80 & 1.59 & 0.39 & 0.73 & 0.81 & 0.71 \\
Sensitivity & 0.48 & 0.80 & 0.71 & 0.81 & 0.68 & 0.74 & 0.71 & 0.77 \\
Specificity & 0.74 & 0.68 & 0.84 & 0.68 & 0.68 & 0.89 & 0.89 & 0.89 \\
\hline
\end{tabular}

AUC of ROC (see Fig. 6), cutoff, sensitivity, and specificity values are presented

demonstrated higher linear correlations with CK-derived metrics for high-grade glioma and comparable sensitivity and specificity in glioma differentiation between low- and highgrade gliomas. In turn, the scalar diffusion metrics based on GK approach exhibited brighter tumour and peritumoural oedema contrasts compared to healthy tissue allowing clinicians to better visualise tumour spreading in a healthy brain tissue environment. Moreover, the heterogeneity rates estimated by RMSE measure are higher in the case of MK, AK, and RK metrics derived from gDKI approach.

The tumour grade differentiation constitutes a challenge for many imaging techniques such as MRI, CT, or PET. This is due to the need of a quantitative parametrisation enabling both the assessment of damage of healthy brain tissue by aggressive growth of tumour and the degree of the malignancy of this process. This is particularly complicated due to multifarious forms of tumour manifestation, including cell swelling, microvasculature proliferation, tumour heterogeneity, and presence of necrosis. Thus, the diffusion MR imaging grants two levels of tumour assessment, that is, easy-to-interpret scalar maps and sensitivity of these maps to the micrometre scale of tissue changes. Therefore, diffusion kurtosis imaging offers an excellent tool for probing brain tumour, enabling estimation of the qualitative and quantitative differences between different glioma grades and their dynamics.

The difference between the conventional and generalised kurtosis estimations is defined by a fraction multiplier $\alpha$ and a quadratic kurtosis term in Eq. (2). It is interesting that dispersion in the distribution between DTI metrics (see Fig. 3) is quite low and does not depend on glioma grade. However, in the case of kurtosis scalar maps (see Fig. 4), in particular for MK and RK, there is a stronger value spreading in low grade glioma metric correlations. Such kind of distribution behaviour can indicate two different types of tissue organisation present at early stages of the glioma tumour: resting healthy brain tissue and highly heterogeneous tumour. As glioma malignancy increases, the heterogeneous tumour tissue becomes dominant due to aggressive growth which leads to higher correlations between $\mathrm{CK}$ and GK metrics, i.e. quadratic kurtosis term might drive this effect. We see that GK-derived MK and RK metrics are more sensitive
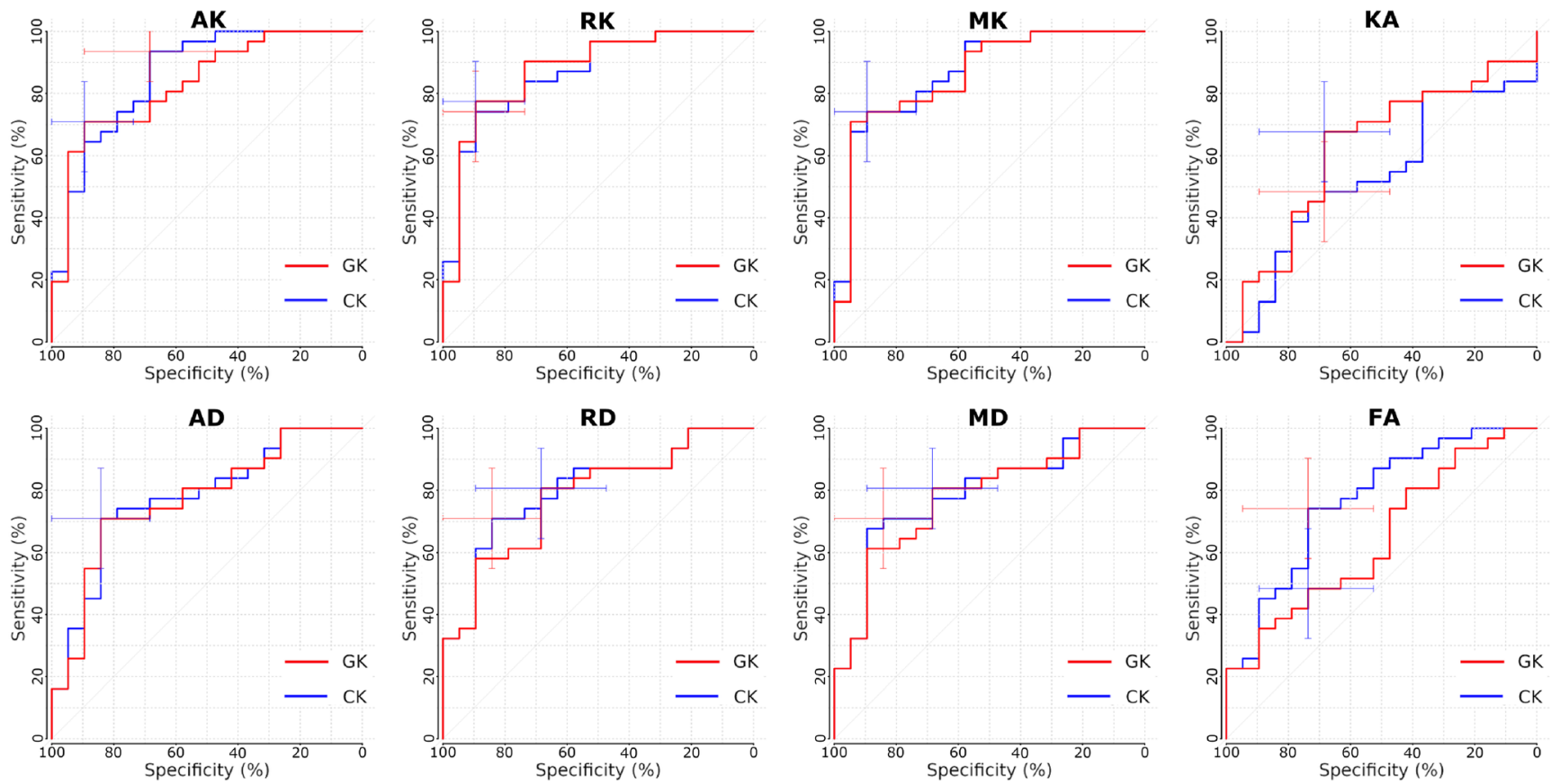

Fig. 6 Receiver operating curves (ROC) for the conventional (CK) and generalised kurtosis (GK) approaches for differentiation of low- and high-grade gliomas. The error bars exhibits the detected sensitivity and specificity of both methods 
Table 4 The Spearman's correlation coefficients $r$ between Ki-67 and diffusion metrics estimated by CK and GK approaches

\begin{tabular}{|c|c|c|c|c|c|c|c|c|}
\hline & FA & $\mathrm{MD}$ & $\mathrm{AD}$ & $\mathrm{RD}$ & $\mathrm{KA}$ & MK & $\mathrm{AK}$ & RK \\
\hline \multicolumn{9}{|l|}{$r$ (all) } \\
\hline $\mathrm{CK}$ & -0.17 & 0.21 & 0.17 & 0.21 & 0.02 & -0.11 & -0.05 & -0.14 \\
\hline GK & -0.19 & 0.22 & 0.17 & 0.23 & 0.01 & -0.07 & -0.03 & -0.08 \\
\hline \multicolumn{9}{|c|}{$r(\mathrm{HGG})$} \\
\hline $\mathrm{CK}$ & 0.22 & -0.34 & -0.30 & -0.30 & 0.01 & 0.42 & 0.31 & 0.50 \\
\hline GK & 0.09 & -0.29 & -0.25 & -0.29 & -0.12 & 0.43 & 0.40 & 0.58 \\
\hline \multicolumn{9}{|c|}{$r(\mathrm{LGG})$} \\
\hline $\mathrm{CK}$ & -0.35 & -0.17 & -0.12 & -0.17 & -0.46 & 0.23 & 0.31 & 0.13 \\
\hline GK & -0.33 & -0.13 & -0.16 & -0.07 & -0.33 & 0.21 & 0.24 & 0.16 \\
\hline
\end{tabular}

to the tumour heterogeneity in low-grade glioma. Therefore, a variation in data dispersion between CK and GK metrics, in particular, in kurtosis metrics, might provide us with an additional information about glioma grades. Notably, this information is accessible only for voxel-wise assessment, where typical glioma grade differentiation is performed for region-averaged diffusion metrics.

This hypothesis is supported by significant differences between CK and GK metrics for kurtosis metrics in contrast to the DTI ones (see Fig. 5). For kurtosis metrics, the significant differences between CK and GK derived values were found in all regions: tumour, oedema, and NAWM. On the other hand, DTI metrics demonstrated significant differences only for normally appearing brain tissue, likely due to the different regions of the brain chosen as NAWM and strong age dependence of the patients. Thus, the quadratic kurtosis term strongly influences the kurtosis scalar metrics while it does not affect the DTI ones.
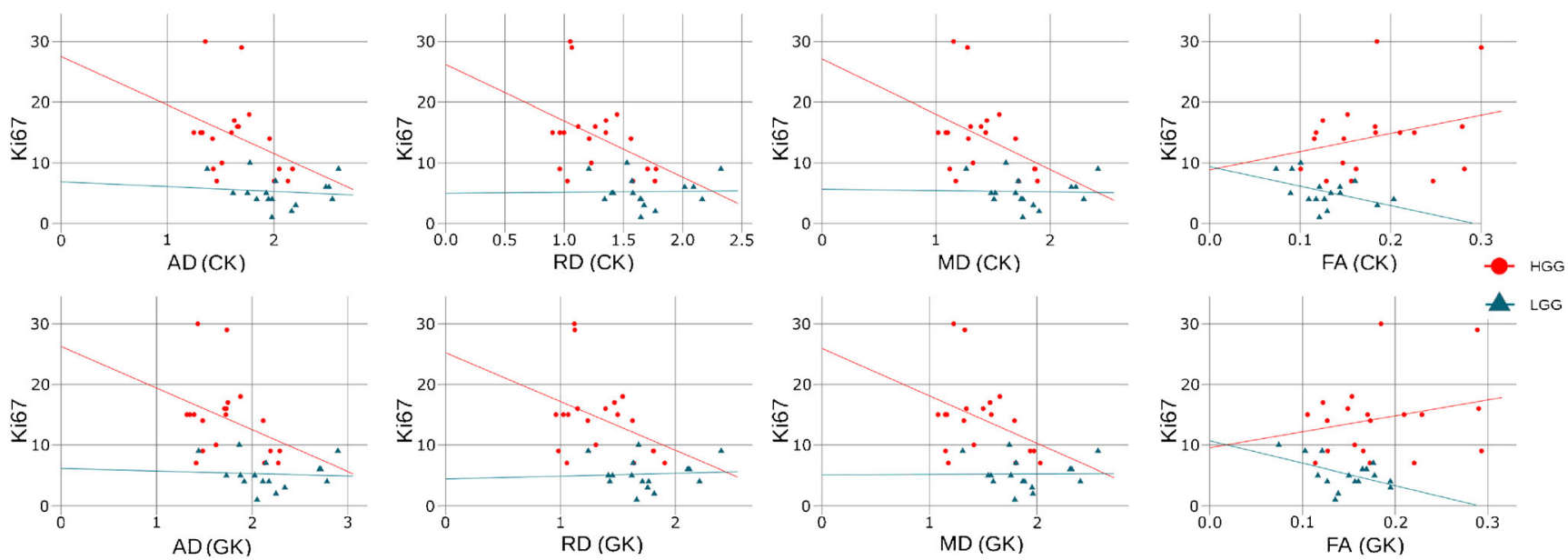

Fig. 7 Correlation plots between Ki-67 and DTI metrics derived from CK and GK approaches. For each glioma grade group (low-grade LGG; highgrade $\mathrm{HGG})$, we plotted the linear regression lines 

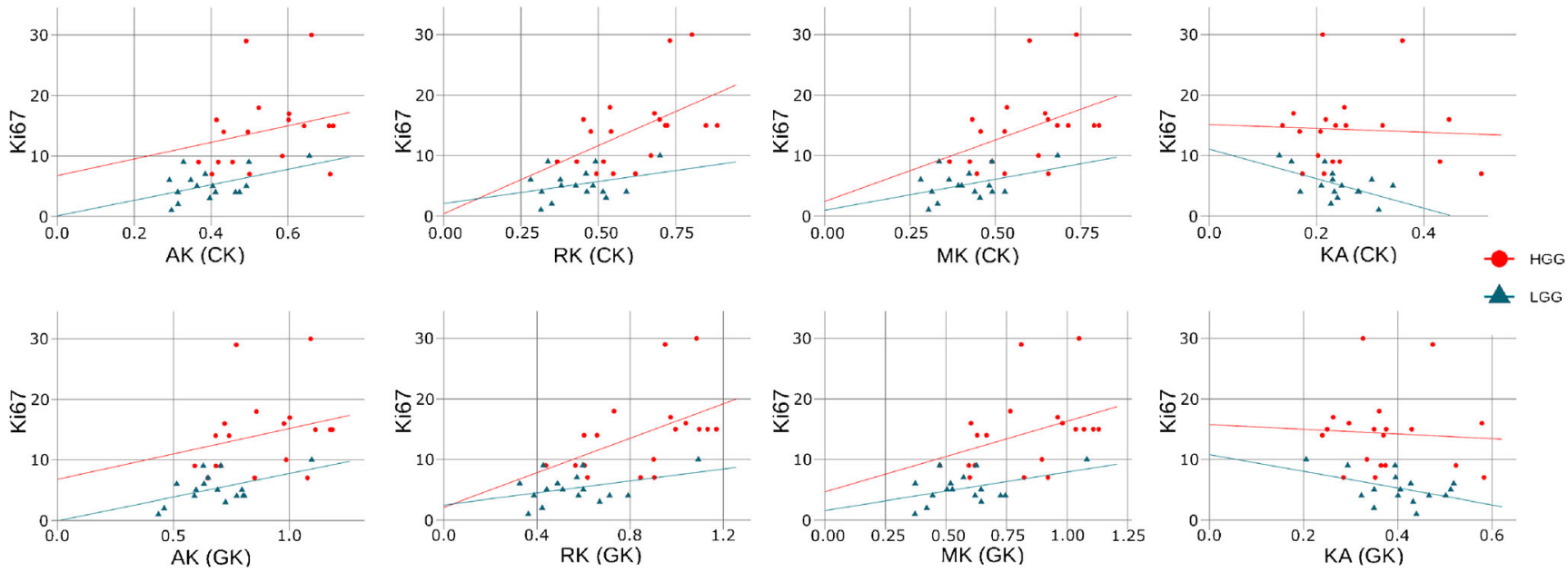

Fig. 8 Correlation plots between Ki-67 and DKI metrics derived from CK and GK approaches. For each glioma grade group (low-grade LGG; highgrade HGG), we plotted the linear regression lines

Our results showed the low correlations between proliferation rate Ki-67 and diffusion metrics (see Figs. 7 and 8) for all subjects. In the case of DTI metrics, we found that diffusion metrics obtained for high-grade gliomas exposed a moderate Ki-67 correlation rate in contrast to the metrics from lowgrade gliomas. In turn, DKI metrics for both CK and GK approaches exhibited the high correlations with Ki-67 rate independently on the glioma grade. Encouraging, our findings of the Ki-67 correlations are in agreement with the previously published works [9, 34, 35].

As a limitation of the study, we should note not an optimal choice of adjusting variable $\alpha$. A more accurate model fitted to the tumour microstructure [36] might increase feasibility of GK approach and its performance in the case of glioma grading. Additionally, advanced diffusion techniques including fast kurtosis [37], isotropic diffusion weighting [38-40], and multidimensional diffusion imaging [41, 42] might help clinicians to perform a robust, fast, and non-invasive glioma differentiation. We plan to implement and apply these approaches for research and patient treatment in the future.

In conclusion, the generalised diffusion kurtosis imaging presents an additional source of information enabling differentiation of low- and high-grade gliomas at the same level as the conventional DKI. The GK approach exhibited higher
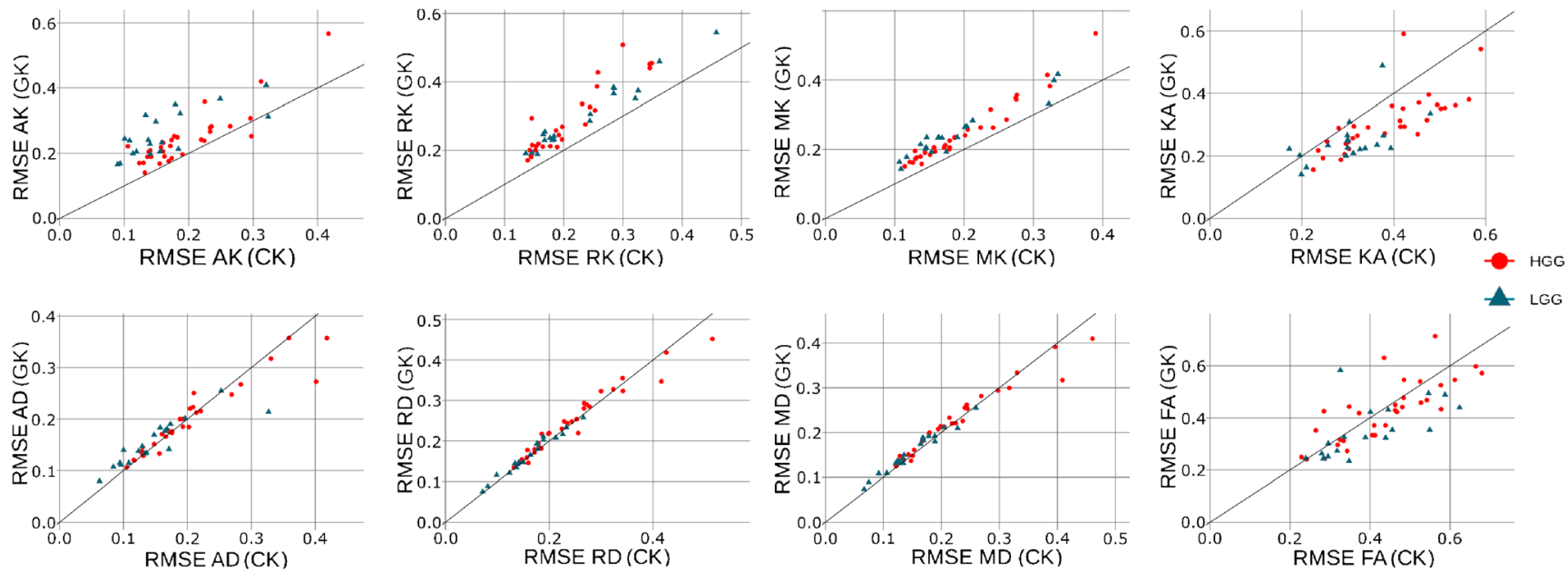

Fig. 9 Scatterplots of root-mean-square estimators (RMSE) computed for CK and GK approaches. The RMSE rates were computed for the solid tumour regions only 
tissue contrast and, thus, offers more sensitive scalar maps to glioma tissue heterogeneity.

Acknowledgement Authors gratefully thank Dr Natalia Tesli for her critical proofreading of the manuscript.

Funding This work was partially supported by Research Council of Norway (249795) and by Russian Foundation for Basic Research (1829-01018)

\section{Compliance with ethical standards}

Conflict of interest Authors declare that they have no conflict of interest.

Ethical approval All procedures performed in study involving human participants were in accordance with the ethical standards of the institutional and/or national research committee and with the 1964 Helsinki declaration and its later amendments or comparable ethical standards.

Informed consent Informed consent was obtained from all individual participants included in the study.

Open Access This article is licensed under a Creative Commons Attribution 4.0 International License, which permits use, sharing, adaptation, distribution and reproduction in any medium or format, as long as you give appropriate credit to the original author(s) and the source, provide a link to the Creative Commons licence, and indicate if changes were made. The images or other third party material in this article are included in the article's Creative Commons licence, unless indicated otherwise in a credit line to the material. If material is not included in the article's Creative Commons licence and your intended use is not permitted by statutory regulation or exceeds the permitted use, you will need to obtain permission directly from the copyright holder. To view a copy of this licence, visit http://creativecommons.org/licenses/by/4.0/.

\section{References}

1. Ostrom QT, Gittleman H, Truitt G, Boscia A, Kruchko C, Barnholtz-Sloan JS (2018) CBTRUS statistical report: primary brain and other central nervous system tumors diagnosed in the United States in 2011-2015. Neuro-Oncology 20(suppl_4):iv1iv86. https://doi.org/10.1093/neuonc/noy131

2. Wesseling P, Capper D (Feb. 2018) WHO 2016 Classification of gliomas. Neuropathol Appl Neurobiol 44(2):139-150. https://doi. org/10.1111/nan.12432

3. N. Upadhyay and A. D. Waldman, "Conventional MRI evaluation of gliomas," The British Journal of Radiology, vol. 84, no. special_issue_2, pp. S107-S111, 2011, https://doi.org/10.1259/ bjr/65711810

4. Wang Q, Zhang H, Zhang JS, Wu C, Zhu WJ, Li FY, Chen XL, Xu BN (2016) The diagnostic performance of magnetic resonance spectroscopy in differentiating high-from low-grade gliomas: a systematic review and meta-analysis. Eur Radiol 26(8):2670-2684. https://doi.org/10.1007/s00330-015-4046-Z

5. Puig J, Blasco G, Schlaug G, Stinear CM, Daunis-i-Estadella P, Biarnes C, Figueras J, Serena J, Hernández-Pérez M, AlberichBayarri A, Castellanos M, Liebeskind DS, Demchuk AM, Menon BK, Thomalla G, Nael K, Wintermark M, Pedraza S (Apr. 2017) Diffusion tensor imaging as a prognostic biomarker for motor recovery and rehabilitation after stroke. Neuroradiology 59(4): 343-351. https://doi.org/10.1007/s00234-017-1816-0

6. Andica $\mathrm{C}$ et al (2019) MR biomarkers of degenerative brain disorders derived from diffusion imaging. Journal of Magnetic Resonance Imaging. https://doi.org/10.1002/jmri.27019

7. Kremneva EI et al (2019) Feasibility of non-Gaussian diffusion metrics in chronic disorders of consciousness. Brain Sciences 9(5):123. https://doi.org/10.3390/brainsci9050123

8. Basser PJ, Mattiello J, LeBihan D (1994) MR diffusion tensor spectroscopy and imaging. Biophys J 66(1):259-267. https://doi. org/10.1016/S0006-3495(94)80775-1

9. Jiang R, Du F-Z, He C, Gu M, Ke Z-W, Li J-H (2014) The value of diffusion tensor imaging in differentiating high-grade gliomas from brain metastases: a systematic review and meta-analysis. PLoS ONE 9(11):e112550. https://doi.org/10.1371/journal.pone. 0112550

10. Svolos P et al (2014) The role of diffusion and perfusion weighted imaging in the differential diagnosis of cerebral tumors: a review and future perspectives. Cancer Imaging 14(1):20. https://doi.org/ $10.1186 / 1470-7330-14-20$

11. Suh CH, Kim HS, Jung SC, Kim SJ (Jul. 2018) Diffusion-weighted imaging and diffusion tensor imaging for differentiating high-grade glioma from solitary brain metastasis: a systematic review and meta-analysis. Am J Neuroradiol 39(7):1208-1214. https://doi.org/10. 3174/ajnr.A5650

12. Jensen JH, Helpern JA, Ramani A, Lu H, Kaczynski K (2005) Diffusional kurtosis imaging: the quantification of non-Gaussian water diffusion by means of magnetic resonance imaging. Magn Reson Med 53(6):1432-1440. https://doi.org/10.1002/mrm.20508

13. Kiselev VG (2017) Fundamentals of diffusion MRI physics: fundamentals of diffusion MRI physics. NMR in Biomedicine 30(3): e3602. https://doi.org/10.1002/nbm.3602

14. Jensen JH, Helpern JA (2010) MRI quantification of non-Gaussian water diffusion by kurtosis analysis. NMR Biomed 23(7):698-710. https://doi.org/10.1002/nbm.1518

15. Rosenkrantz AB, Padhani AR, Chenevert TL, Koh DM, de Keyzer F, Taouli B, le Bihan D (2015) Body diffusion kurtosis imaging: basic principles, applications, and considerations for clinical practice: body diffusion kurtosis imaging. J Magn Reson Imaging 42(5):1190-1202. https://doi.org/10.1002/jmri.24985

16. Grinberg F, Maximov II, Farrher E, Neuner I, Amort L, Thönneßen H, Oberwelland E, Konrad K, Shah NJ (2017) Diffusion kurtosis metrics as biomarkers of microstructural development: a comparative study of a group of children and a group of adults. NeuroImage 144:12-22. https://doi.org/10.1016/j.neuroimage.2016.08.033

17. Hempel J-M, Schittenhelm J, Brendle C, Bender B, Bier G, Skardelly M, Tabatabai G, Castaneda Vega S, Ernemann U, Klose U (2017) Histogram analysis of diffusion kurtosis imaging estimates for in vivo assessment of 2016 WHO glioma grades: a cross-sectional observational study. Eur J Radiol 95:202-211. https://doi.org/10.1016/j.ejrad.2017.08.008

18. Tietze A, Hansen MB, Østergaard L, Jespersen SN, Sangill R, Lund TE, Geneser M, Hjelm M, Hansen B (2015) Mean diffusional kurtosis in patients with glioma: initial results with a fast imaging method in a clinical setting. Am J Neuroradiol 36(8):1472-1478. https://doi.org/10.3174/ajnr.A4311

19. Raab P, Hattingen E, Franz K, Zanella FE, Lanfermann H (2010) Cerebral Gliomas: diffusional kurtosis imaging analysis of microstructural differences. Radiology 254(3):876-881. https://doi.org/ 10.1148/radiol.09090819

20. Falk Delgado A, Nilsson M, van Westen D, Falk Delgado A (2018) Glioma grade discrimination with MR diffusion kurtosis imaging: a meta-analysis of diagnostic accuracy. Radiology 287(1):119-127. https://doi.org/10.1148/radiol.2017171315 
21. Maximov II, Tonoyan AS, Pronin IN (2017) Differentiation of glioma malignancy grade using diffusion MRI. Physica Medica 40: 24-32. https://doi.org/10.1016/j.ejmp.2017.07.002

22. Qi X-X, Shi DF, Ren SX, Zhang SY, Li L, Li QC, Guan LM (2018) Histogram analysis of diffusion kurtosis imaging derived maps may distinguish between low and high grade gliomas before surgery. Eur Radiol 28(4):1748-1755. https://doi.org/10.1007/s00330-0175108-1

23. Vellmer S, Tonoyan AS, Suter D, Pronin IN, Maximov II (2018) Validation of DWI pre-processing procedures for reliable differentiation between human brain gliomas. Z Med Phys 28(1):14-24. https://doi.org/10.1016/j.zemedi.2017.04.005

24. Jensen JH, Mohanty V, McKinnon ET, Helpern JA (2017) Optimizing the signal model for diffusional kurtosis imaging. In: Proceedings of International Society of Magnetic Resonance in Medicine, 22-27 April, Honolulu, USA, p 1731 [Online]. Available: http://indexsmart.mirasmart.com/ISMRM2017/ PDFfiles/1731.html

25. Feiden S, Feiden W (2008) WHO-Klassifikation der ZNSTumoren: Revidierte Fassung von 2007 mit kritischen Anmerkungen zum "Typing" und "Grading" diffuser Gliome. Pathologe 29(6):411-421. https://doi.org/10.1007/s00292-0081064-5

26. Maximov II, Alnæs D, Westlye LT (2019) Towards an optimised processing pipeline for diffusion magnetic resonance imaging data: effects of artefact corrections on diffusion metrics and their age associations in UK Biobank. Hum Brain Mapp 40(14):41464162. https://doi.org/10.1002/hbm.24691

27. Veraart J, Novikov DS, Christiaens D, Ades-aron B, Sijbers J, Fieremans E (2016) Denoising of diffusion MRI using random matrix theory. NeuroImage 142:394-406. https://doi.org/10.1016/ j.neuroimage.2016.08.016

28. Kellner E, Dhital B, Kiselev VG, Reisert M (2016) Gibbs-ringing artifact removal based on local subvoxel-shifts: Gibbs-ringing artifact removal. Magn Reson Med 76(5):1574-1581. https://doi.org/ $10.1002 / \mathrm{mrm} .26054$

29. Jenkinson M, Beckmann CF, Behrens TEJ, Woolrich MW, Smith SM (2012) FSL. NeuroImage 62(2):782-790. https://doi.org/10. 1016/j.neuroimage.2011.09.015

30. Andersson JLR, Skare S, Ashburner J (2003) How to correct susceptibility distortions in spin-echo echo-planar images: application to diffusion tensor imaging. NeuroImage 20(2):870-888. https:// doi.org/10.1016/S1053-8119(03)00336-7

31. Andersson JLR, Sotiropoulos SN (2016) An integrated approach to correction for off-resonance effects and subject movement in diffusion MR imaging. NeuroImage 125:1063-1078. https://doi.org/10. 1016/j.neuroimage.2015.10.019

32. Veraart J, Sijbers J, Sunaert S, Leemans A, Jeurissen B (2013) Weighted linear least squares estimation of diffusion MRI parameters: strengths, limitations, and pitfalls. NeuroImage 81: 335-346. https://doi.org/10.1016/j.neuroimage.2013.05.028

33. Diedenhofen B, Musch J (2015) Cocor: a comprehensive solution for the statistical comparison of correlations. PLoS One 10: E0131499. https://doi.org/10.1371/journal.pone.0121945

34. Zhang J, Chen X, Chen D, Wang Z, Li S, Zhu W (2018) Grading and proliferation assessment of diffuse astrocytic tumors with monoexponential, biexponential, and stretched-exponential diffusion-weighted imaging and diffusion kurtosis imaging. Eur $\mathrm{J}$ Radiol 109:188-195. https://doi.org/10.1016/j.ejrad.2018.11.003

35. Zhao J, Wang YL, Li XB, Hu MS, Li ZH, Song YK, Wang JY, Tian YS, Liu DW, Yan X, Jiang L, Yang ZY, Chu JP (2019) Comparative analysis of the diffusion kurtosis imaging and diffusion tensor imaging in grading gliomas, predicting tumour cell proliferation and IDH-1 gene mutation status. J Neuro-Oncol 141:195-203. https://doi.org/10.1007/ s11060-018-03025-7

36. Zaccagna F, Riemer F, Priest AN, McLean MA, Allinson K, Grist JT, Dragos C, Matys T, Gillard JH, Watts C, Price SJ, Graves MJ, Gallagher FA (Oct. 2019) Non-invasive assessment of glioma microstructure using VERDICT MRI: correlation with histology. Eur Radiol 29(10):5559-5566. https://doi.org/10.1007/s00330-0196011-8

37. Hansen B, Lund TE, Sangill R, Stubbe E, Finsterbusch J, Jespersen SN (2016) Experimental considerations for fast kurtosis imaging: experimental considerations for fast kurtosis imaging. Magn Reson Med 76(5):1455-1468. https://doi.org/10.1002/mrm.26055

38. Eriksson S, Lasic S, Topgaard D (2013) Isotropic diffusion weighting in PGSE NMR by magic-angle spinning of the q-vector. J Magn Reson 226:13-18. https://doi.org/10.1016/j.jmr.2012.10. 015

39. Vellmer S, Stirnberg R, Edelhoff D, Suter D, Stöcker T, Maximov II (2017) Comparative analysis of isotropic diffusion weighted imaging sequences. J Magn Reson 275:137-147. https://doi.org/10. 1016/j.jmr.2016.12.011

40. Maximov II, Vellmer S (2019) Isotropically weighted intravoxel incoherent motion brain imaging at $7 \mathrm{~T}$. Magn Reson Imaging 57: 124-132. https://doi.org/10.1016/j.mri.2018.11.007

41. Topgaard D (2017) Multidimensional diffusion MRI. J Magn Reson 275:98-113. https://doi.org/10.1016/j.jmr.2016.12.007

42. Westin C-F, Knutsson H, Pasternak O, Szczepankiewicz F, Özarslan E, van Westen D, Mattisson C, Bogren M, O'Donnell LJ, Kubicki M, Topgaard D, Nilsson M (2016) Q-space trajectory imaging for multidimensional diffusion MRI of the human brain. NeuroImage 135:345-362. https://doi.org/10.1016/j.neuroimage. 2016.02.039

Publisher's note Springer Nature remains neutral with regard to jurisdictional claims in published maps and institutional affiliations. 